Proceedings of the 6th Polish Symposium of Physics in Economy and Social Sciences (FENS2012), Gdańsk, Poland

\title{
Spectral Analysis of Capital Markets
}

\author{
A. Dyka, P. DudojĆ* And J. Garus \\ Gdańsk University of Technology, G. Narutowicza 11/12, 80-233 Gdańsk, Poland
}

\begin{abstract}
In this paper the problem of cycles existence in capital markets is addressed. A spectral analysis algorithm, which reduces signal-to-noise ratio, is proposed to derive cycle periodograms for the yield function of DJIA, WIG 20, and NIKKEI 225 indices. Peaks of the the periodograms provide premises to postulate the existence of some possible cycles. The 3.5 year periodicity in all 3 indices, which can be related to the Kitchin cycle is found to be the most distinctive one.
\end{abstract}

DOI: 10.12693 /APhysPolA.123.518

PACS: 89.65.Gh, 02.30.Nw, 02.60.Ed, 89.90.+n

\section{Introduction}

The problem of significant importance in capital markets is the confirmation or denial of the cycles (periodicity) existence. In this paper the attempt, to show that some periodicity in markets can be found, is made. A certain concept of spectral analysis using linear filtering theory has been here proposed, and used to identify possible cycles in DJIA, WIG 20 (Warsaw Stock Exchange index), and NIKKEI 225. Spectral analysis has extensively been used in various areas of technology and science including economics [1, 2]. Research on World GDP dynamics is a suitable example of standard spectral analysis application in economics [3].

The outcome of the spectral analysis here proposed has the form of a periodogram. Peaks of the periodogram may indicate the appearance of cycles. However, due to possible nonlinearities in market phenomena, and limitations of the spectral analysis some peaks can be spurious ones. Therefore, conclusions concerning identification of possible cycles should always be carefully revised on the case by case basis. The spectral analysis here presented enables us to postulate the 3.5 year periodicity appearance in all indices, which believeably can be interpreted as the Kitchin cycle [4]. Some other possible cycles are also suggested.

\section{Introduction to linear filtering theory}

For the purpose of our research the formalism of linear filtering theory, briefly presented below, has been used. This approach is very useful in simplifying and expediting computations, as well as for explaining and interpreting results.

In the general case, linear differential equations with constant coefficients can be used to describe linear systems [5]. The condition of linearity implies the frequency preservation property [6]. It says that the response of the filter contains only these frequencies which are present in the input signal.

*corresponding author; e-mail: pdudojcœ๐2.p1
Every linear system can be described by a corresponding time domain function called the impulse response $h(t)$, which is defined as the system response to the Dirac delta function applied to the input. Impulse response is a unique representation of a filter in the time domain (Fig. 1).

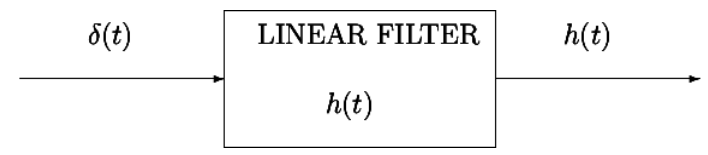

Fig. 1. Definition of filter impulse response $h(t)$.

The relationship between input and output signal of a linear system is described by the filtering equation, which takes the following form (see Fig. 2):

$$
y(t)=x(t) * h(t),
$$

where $x(t)$ is the input signal, $y(t)$ is the output signal and the symbol $*$ denotes convolution, which is defined, as follows:

$$
y(t)=\int_{-\infty}^{\infty} h(\tau) x(t-\tau) \mathrm{d} \tau .
$$

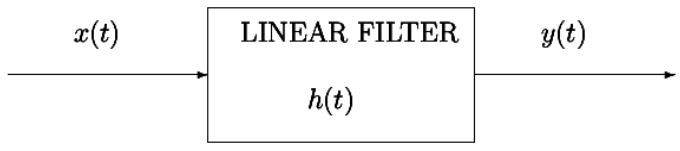

Fig. 2. The general representation of a linear filter.

This equation defines the linear filtering in the most general way.

In the discrete time domain, an impulse response, limited in the argument, is called the finite impulse response (FIR).

\section{Assumptions and definitions}

Our experience on analyses of markets indicates that one should seek a periodicity in the yield function (defined below), rather than in index quotations. 
Let us assume a market quotation $x(p)$ of length $q$ :

$x(p), \quad p=1,2, \ldots q$.

Then, the logarithmic return or yield function can be defined as follows:

$$
y(p, n)=\log (x(p+n))-\log (x(p)),
$$

where $n$ takes value from 1 to $q$ and represents the duration of the investment. Therefore, by computing the above function, defined by (3.2), the input sequence of quotations (3.1) can be transformed into the following sequence, called from now on, the yield function

$$
y f_{n}(i), \quad i=1,2, \ldots q-n+1 \text {. }
$$

In terms of linear filtering theory, the computation of yield function (3.3) is equivalent to operation of linear filtering of the $\log (x(p))$ with the specific FIR filter. The impulse response of this filter is given by the odd pair of impulses [7], which should have the following form (Fig. 3):

$$
h_{n}(p)=\delta(p+n)-\delta(p) .
$$

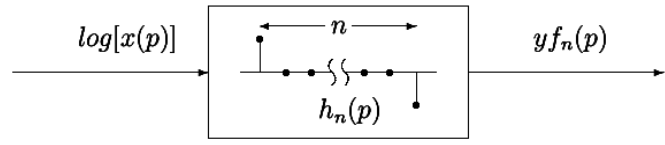

Fig. 3. Impulse response $h_{n}(p)$ for computing yield function $y f_{n}(p)$.

Having applied the above filtering to the log of quotations one can obtain the yield function for an arbitrary duration of the investment, defined by $n$.

\section{Concept of spectral analysis}

The concept of the spectral analysis, here proposed, is shown in Fig. 4. Assume that the input quotations have already been transformed into the yield function $y f_{n}(p)$. Then, the yield function is divided into adjacent subintervals of the duration $n$, as shown in Fig. 4a. These subintervals are averaged as shown in Fig. 4b. The number of subintervals equals $k$ as follows:

$$
k=\left\lfloor\left(\frac{l}{n}\right)\right\rfloor,
$$

where:

- $l$ is duration of the yield function,

- $\lfloor\cdot\rfloor$ stands for the operation of rounding to the largest integer in the value of $l / n$.

After averaging we have the function $f(p)$ of the duration $n$, which is subsequently used to determine the existence of a cycle of the period equal to $n$.

Averaging, in the ideal case, reduces signal-to-noise ratio by the factor of square root of $k[6]$. After averaging a standard procedure of computing first harmonic for $f(p)$ is applied. a)
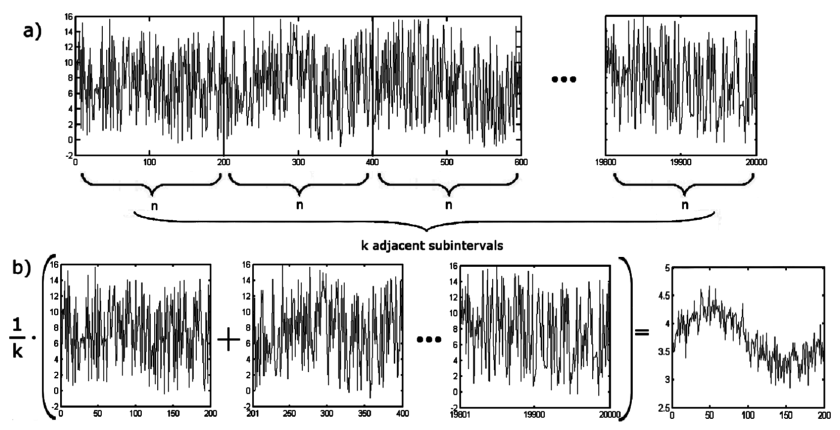

Fig. 4. The idea of spectral analysis. (a) Chopping yield function into $k$ adjacent subintervals. (b) Computing the average average $f(p)$, which reduces signal to noise ratio.
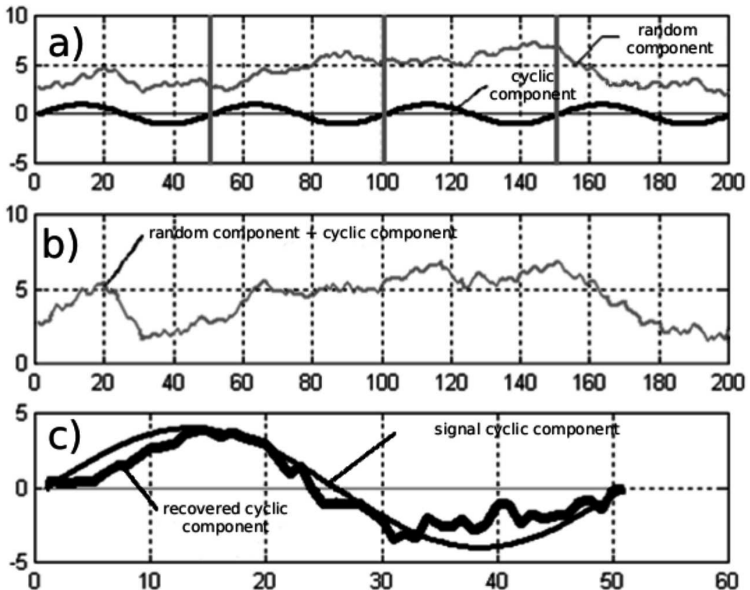

Fig. 5. Recovery of cyclic component using simulated data. (a) Random and cyclic component. (b) Superposition of random and cyclic component. (c) Original and recovered cyclic component.

The idea of identifying periodicity, by means of this concept, was tested first using some simulated data as shown in Fig. 5. In Fig. 5a we have two components of the duration 200, i.e., cyclic with four periods of 50 samples and a random component. In Fig. $5 \mathrm{~b}$ we have a composite signal that is random component added to cyclic component, whereas in Fig. $5 \mathrm{c}$ we have the result of recovery of the cyclic component from the composite signal, using the described concept of spectral analysis.

\section{Results}

Consequently, the spectral analysis algorithm has been applied to real data, as follows:

1. DJIA, quoted from May 26, 1896 to December 30, 2011, [8]

2. WIG 20 (Warsaw Stock Exchange index), quoted from April 16, 1994 to December 30, 2011, [9] 
3. NIKKEI 225, quoted from January 4, 1984 to December 30, 2011, [10].

For all the indices the magnitude of the first harmonic coefficient vs. $n$ have been computed in the form of periodogram. In Fig. 6 the periodogram obtained for the yield function of DJIA index is shown for $n=3, \ldots, 3999$. A number of cycles has been observed.

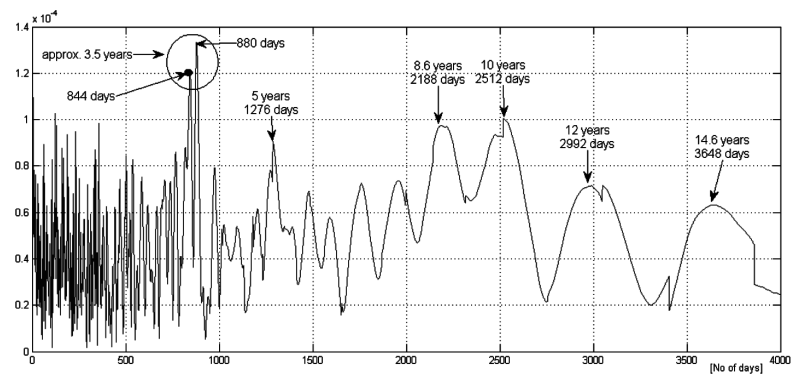

Fig. 6. Periodogram for DJIA index yield function for $n=3, \ldots, 3999$.

The cycles that can be observed in the DJIA yield function have periods lengths close to 3.5 years (844 days), 5 years (1276 days), 8.6 years (2188 days), 10 years ( 2512 days), 12 years (2992 days), and 14.6 years (3648 days). The strongest peak observed is close to 3.5 years period.

The periodogram for WIG 20 index yield function is presented in Fig. 7. It was observed that WIG 20 yield function has cycle lengths close to 1 year (232 days), 2 years (492 days), 2.5 years (640 days), and 3.5 years (844 days).

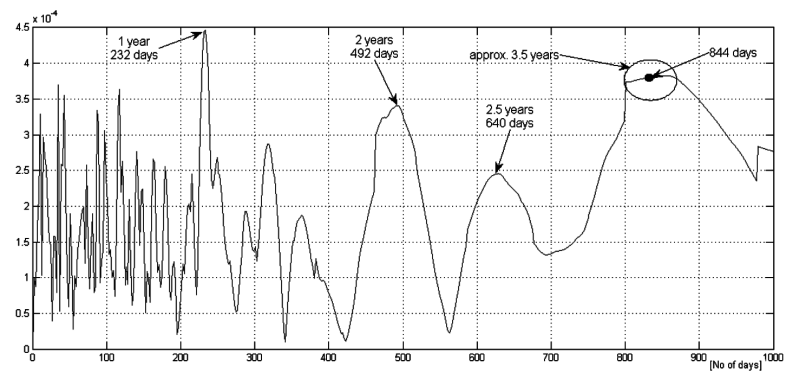

Fig. 7. Periodogram for WIG 20 index yield function for $n=3, \ldots, 1000$.

The periodogram for NIKKEI 225 is shown in Fig. 8. In this case the most distinctive cycles can be observed for periods lengths of approximately 2 years (504 days), 2.5 years (640 days), 3.5 years ( 832 days), and 5 years (1348 days).

Apart from that some other minor cycles can be observed in all analyzed indices.

Due to different lengths of quotations varying from 4400 for the WIG 20 to almost 29000 in the case of DJIA, it is rather unrealistic to expect that the peaks of periodograms will overlap absolutely. The other cause for the discrepancy between periodogram peaks is the

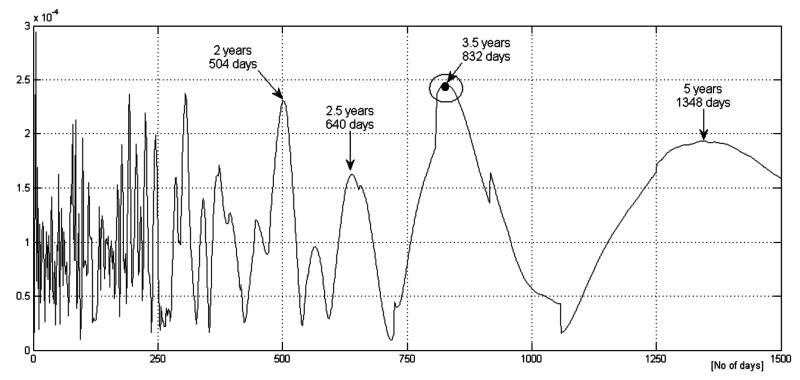

Fig. 8. Periodogram for NIKKEI 225 index yield function for $n=3, \ldots, 1500$.

varying number of business days in every country, and every year. For that reason we decided to analyze periodograms separately (without formal correlating them), to find similarities beetwen them by visual inspection. We found that the 3.5 years cycle appears in the case of all studied indices, as follows: 844 days, 844 days, and 832 days for DJIA, WIG 20, and NIKKEI 225, respectively. Moreover, we made a quick check with the Hang Seng index which has also shown the 3.5 years cycle on the periodogram. Therefore, we can postulate that the most distinctive 3.5 year periodicity can be interpreted as the manifestation of the Kitchin cycle [4]. We can also find that $2,2.5$, and 5 years cycles can be observed distinctively in 2 out of 3 analysed indices. However, the latter statement should be considered as an initial conclusion, which needs to be confirmed by further research.

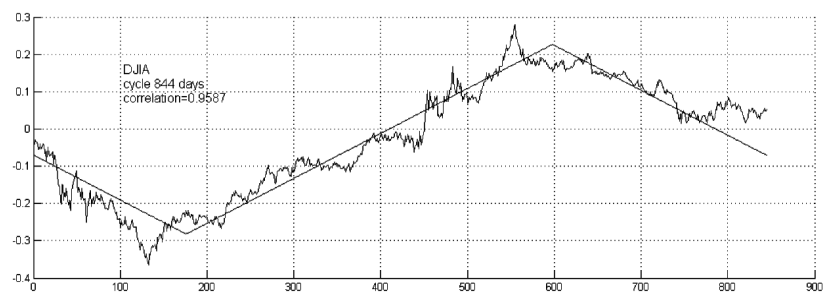

Fig. 9. Adjustment of 844 days cycle to the last subinterval of DJIA yield function (last quotation on December 30,2011$)$.

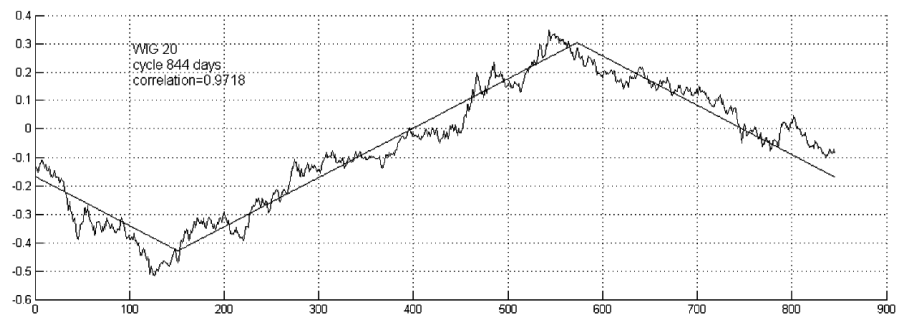

Fig. 10. Adjustment of 844 days cycle to the last subinterval of WIG 20 yield function (last quotation on December 30, 2011). 


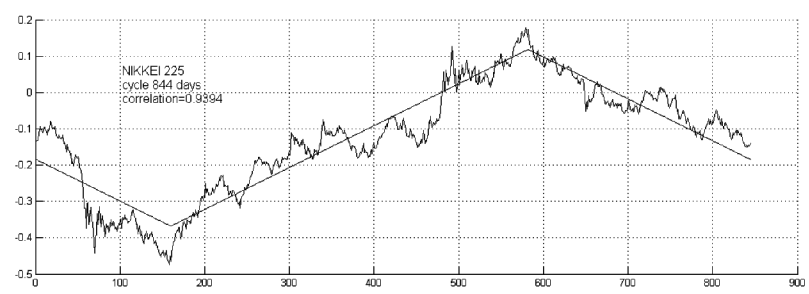

Fig. 11. Adjustment of 844 days cycle to the last subinterval of NIKKEI 225 yield function (last quotation on December 30, 2011).

Eventually, using the Pearson correlation coefficient, we adjusted the 844 days ( 3.5 year) cycle to the last subinterval of the yield function of all analyzed indices, for the last quotation on December 30, 2011. This coefficient takes the value as follows: $0.9587,0.9718$, and 0.9394 for DJIA, WIG 20, and NIKKEI 225, respectively. The results of the adjustment are shown in Figs. 9-11 for DJIA, WIG 20, and NIKKEI 225, respectively.

\section{Concluding remarks}

The proposed method of spectral analysis, applied to the yield function of the three indices, i.e. DJIA, WIG 20, and NIKKEI 225, has provided preliminary promising results. The most distinctive, common for all indices, 3.5 year periodicity can be related to the Kitchin cycle. Other common periodicities namely $2,2.5$, and 5 years can also be observed. However, the latter result, as well as the "predictive power" of the identified cycles needs to be confirmed by a further research.

\section{References}

[1] A. Tacobucci, in: New Tools of Economics Dynamics, Eds. J. Leskow, L.F. Punzo, M.P. Anyul, Springer, Berlin 2005, p. 203.

[2] P. Stoica, Introduction to Spectral Analysis, Prentice Hall, Upper Saddle River 1997.

[3] A.V. Korotayev, S.V. Tsirel, Structure and Dynamics 4, 3 (2010).

[4] J. Kitchin, Rev. Econom. Statist. 5, 10 (1923).

[5] R.A. Gabel, Signals and Linear Systems, John Wiley and Sons, New York 1973.

[6] P.A. Lynn, An Introduction to the Analysis and Processing of Signals, Halsted Press, New York 1973.

[7] R. Bracewell, The Fourier Transform and Its Applications, McGraw-Hill, New York 1965.

[8] DJIA quotations: www.research.stlouisfed.org .

[9] WIG 20 quotations: www. bossa.pl .

[10] NIKKEI 225 quotations: www.finance.yahoo.com . 